\title{
Surface incompressibility from semiclassical relativistic mean field calculations
}

\author{
S. K. Patra, ${ }^{*}$ M. Centelles, X. Viñas, and M. Del Estal \\ Departament d'Estructura i Constituents de la Matèria, Facultat de Física, Universitat de Barcelona, Diagonal 647, \\ E-08028 Barcelona, Spain \\ (Received 31 October 2001; published 11 March 2002)
}

\begin{abstract}
By using the scaling method and the Thomas-Fermi and extended Thomas-Fermi approaches to relativistic mean field theory the surface contribution to the leptodermous expansion of the finite nuclei incompressibility $K_{A}$ has been self-consistently computed. The validity of the simplest expansion, which contains volume, volume-symmetry, surface, and Coulomb terms, is examined by comparing it with self-consistent results of $K_{A}$ for some currently used nonlinear $\sigma-\omega$ parameter sets. A numerical estimate of higher-order contributions to the leptodermous expansion, namely, the curvature and surface-symmetry terms, is made.
\end{abstract}

DOI: 10.1103/PhysRevC.65.044304

PACS number(s): 24.30.Cz, 21.60.-n, 21.30.Fe

The curvature of the nuclear matter equation of state, i.e., the nuclear matter incompressibility $K_{\infty}$ is a key quantity in nuclear physics because it is related to many properties of nuclei (such as radii, masses, and giant resonances), heavyion collisions, neutron stars, and supernova collapses. One important source of information on $K_{\infty}$ is provided by the study of the isoscalar giant monopole resonance (GMR) (breathing mode) in finite nuclei. In the nonrelativistic frame, theoretical microscopic calculations based on the randomphase approximation [1] and approximations to it such as the scaling method [2-4] or constrained calculations [3-5] using Skyrme [3] and Gogny [6] effective forces lead to a nuclear matter incompressibility coefficient $K_{\infty}$ of $215 \pm 15$ $\mathrm{MeV}[6,7]$. A similar analysis carried out within the relativistic mean field (RMF) theory with nonlinear $\sigma-\omega$ effective Lagrangians gives a value of $K_{\infty}$ slightly higher, that is, 250$270 \mathrm{MeV}$ [8].

The nuclear matter incompressibilty $K_{\infty}$ is not a directly measurable quantity; what is measured is, actually, the energy $E_{M}$ of the GMR of finite nuclei. It is convenient to write this energy in terms of the incompressibility $K_{A}$ for a finite nucleus of mass number $A$ as

$$
E_{M}=\sqrt{\frac{\hbar^{2} K_{A}}{M\left\langle r^{2}\right\rangle}}
$$

where $\left\langle r^{2}\right\rangle$ is the rms matter radius and $M$ the nucleon mass. The finite nucleus incompressibilty $K_{A}$ can be parametrized by means of a leptodermous expansion [2] that is similar to the liquid drop mass formula

$$
K_{A}=K_{\infty}+K_{s f} A^{-1 / 3}+K_{v s} I^{2}+K_{c o u l} Z^{2} A^{-4 / 3}+\cdots,
$$

where $I=(N-Z) / A$ is the neutron excess. Equation (2) suggests that it is possible to fit the coefficients of the expansion to the experimental data in a model independent way. Although some effort along these lines has been made in the past [9], the fact that a fit of the parameters of Eq. (2) to experimental data does not lead to a unique determination of

\footnotetext{
*Present address: Institute of Physics, Sachivalaya Marg, Bhubaneswar-751 005, India.
}

the parameters is well established $[6,10,11]$. Rather, the nuclear matter incompressibility has to be determined from effective forces that reproduce, in a microscopic calculation, the experimental values of the GMR excitation energy in heavy nuclei [6].

It is also possible to fit $K_{A}$ calculated microscopically within the scaling model for a given effective interaction to the leptodermous expansion Eq. (2). This has been done, for example, in the nonrelativistic frame using Skyrme forces [12]. In this case the coefficients entering Eq. (2) can be expressed through infinite and semi-infinite nuclear matter properties calculated with the Hartree-Fock approximation for each considered interaction. In particular, the volumesymmetry $\left(K_{v s}\right)$ and Coulomb $\left(K_{\text {coul }}\right)$ coefficients depend on some parameters of the liquid droplet model [13] computed only using nuclear matter properties [2]. The surface coefficient $K_{s f}$, also derived in [2], can be written as [14]

$$
K_{s f}=4 \pi r_{0}^{2}\left[\left(22+\frac{54}{K_{\infty}} \rho_{0}^{3} \ddot{e}_{\infty}\left(\rho_{0}\right)\right) \sigma\left(\rho_{0}\right)+9 \rho_{0}^{2} \ddot{\sigma}\left(\rho_{0}\right)\right] \text {. }
$$

The surface tension $\sigma$ is calculated in symmetric semiinfinite nuclear matter and is defined as

$$
\sigma\left(\rho_{c}\right)=\int_{-\infty}^{+\infty}\left\{\mathcal{H}(\rho)-e_{\infty}\left(\rho_{c}\right) \rho\right\} d z
$$

where $\rho$ is the density profile whose central value is given by $\rho_{c}=\rho(-\infty), \mathcal{H}$ is the energy density, and $e_{\infty}$ is the energy per particle in nuclear matter at density $\rho_{c}$. In Eq. (3) dots indicate the derivatives with respect to the central density and all the quantities are evaluated at a central density equal to the nuclear matter saturation density $\rho_{0}$, which is related to the radius constant $r_{0}$ through $4 \pi r_{0}^{3} \rho_{0} / 3=1$.

The key quantity entering Eq. (3) is $\ddot{\sigma}$, which is the second derivative of $\sigma\left(\rho_{c}\right)$ with respect to $\rho_{c}$ calculated at $\rho_{c}$ $=\rho_{0}$. The determination of $\ddot{\sigma}$ also requires knowledge of how the density profile $\rho$ is modified during compression [15]. In the study of the breathing mode a scaling transformation of the densities is assumed. Actually, the coefficients entering the parametrization (2) can be derived under this 
hypothesis [2]. The scaling transformation means that the density changes according to the transformation $\mathbf{r} \rightarrow \lambda \mathbf{r}$ and consequently

$$
\rho_{\lambda}(\mathbf{r})=\lambda^{3} \rho(\lambda \mathbf{r})
$$

Thus, in the scaling approach

$$
\ddot{\sigma}\left(\rho_{0}\right)=\left[\frac{d^{2} \sigma\left(\rho_{c}\right)}{d \rho_{c}^{2}}\right]_{\rho_{0}}=\frac{1}{9 \rho_{0}^{2}}\left[\frac{d^{2} \sigma}{d \lambda^{2}}\right]_{\lambda=1} .
$$

To obtain the surface incompressibility coefficient $K_{s f}$ for a given effective interaction, it is necessary, first of all, to calculate the scaled surface tension $\sigma_{\lambda}$ by replacing the densities by the scaled densities given by Eq. (5) in Eq. (4). In the nonrelativistic frame this can be easily done within the Hartree-Fock scheme using zero-range Skyrme forces and a simple analytical expression for $\sigma_{\lambda}$ is obtained [12,14].

The self-consistent calculation of $K_{s f}$ within the RMF approximation using the $\sigma-\omega$ model is more involved due to the problem of the change in the meson fields induced by the scaled nuclear densities [16]. To our knowledge, only approximate calculations of $K_{s f}$ have been developed in the past for the relativistic model. This is the case of the relativistic Thomas-Fermi (RTF) calculations of Refs. [16,17] where a local density approximation of the meson fields was used. Another approach is related with the study of nuclei under an external pressure. Starting from a schematic energy density functional and adding a density-dependent constraint that simulates the pressure, analytical expressions for the surface tension $\sigma$ as a function of the bulk density $\rho_{c}$ can be derived for a wide class of compression modes, in particular, for the scaling mode [15]. This way one obtains the following formula for $\ddot{\sigma}$ in the scaling mode

$$
\ddot{\sigma}\left(\rho_{0}\right)=-\frac{19}{81} \frac{K_{\infty} \alpha}{\rho_{0}},
$$

where $\alpha$ is the surface diffuseness parameter of a symmetric Fermi density. This pocket formula has been employed to estimate $K_{s f}$ in the RMF model for several nonlinear $\sigma$ - $\omega$ parameter sets [19]. A symmetric Fermi function that reproduces in the best way the density profile obtained from a Hartree calculation of semi-infinite nuclear matter has been used in Ref. [19] to determine the $\alpha$ parameter of Eq. (7).

Very recently, the scaling method applied to the RMF theory in the RTF and relativistic extended Thomas-Fermi (RETF) approaches has been used to self-consistently obtain the excitation energy of the GMR of finite nuclei [20,21]. Our aim in the present paper is, first to obtain the surface coefficient $K_{s f}$ self-consistently in the RTF and RETF approaches developed in Ref. [20,21] for some linear and nonlinear $\sigma-\omega$ parameter sets. On the other hand, we want to check whether the leptodermous expansion of the finite nucleus incompressibility Eq. (2) can reproduce the corresponding fully self-consistent value obtained in the RETF approach [21] with some selected nonlinear $\sigma$ - $\omega$ parameter sets.
The key point of our semiclassical approach is that the local Fermi momentum $k_{F}$ and the effective mass $m^{*}$ scale as $[20,21]$

$$
k_{F \lambda}=\lambda k_{F}(\lambda \mathbf{r}), \quad m_{\lambda}^{*}(\mathbf{r})=\lambda \tilde{m}^{*}(\lambda \mathbf{r}),
$$

where $\tilde{m}^{*}$ is still a function of $\lambda$. With the help of Eq. (8), the nuclear part of the energy and the scalar density including $\hbar^{2}$ corrections, which are functionals of $k_{F}$ and $m^{*}$, scale as

$$
\mathcal{E}_{\lambda}(\mathbf{r})=\lambda^{4} \widetilde{\mathcal{E}}(\lambda \mathbf{r}), \quad \rho_{s \lambda}(\mathbf{r})=\lambda^{3} \widetilde{\rho}_{s}(\lambda \mathbf{r})
$$

Again $\widetilde{\mathcal{E}}$ and $\widetilde{\rho}_{s}$ are functions of the collective coordinate $\lambda$ because of their dependence on $\widetilde{m}^{*}$. Thus the scaled surface tension can be written as [20-22]

$$
\begin{aligned}
\sigma_{\lambda}= & \int\left[\lambda^{4} \mathcal{H}_{\lambda}(\lambda z)-e_{\infty}\left(\lambda^{3} \rho_{0}\right) \lambda^{3} \rho(\lambda z)\right] \frac{d(\lambda z)}{\lambda} \\
= & \int d(\lambda z)\left\{\lambda^{3} \widetilde{\mathcal{E}}+\lambda^{2} g_{v} V_{\lambda} \rho-\frac{1}{2} \lambda\left[\left(\nabla V_{\lambda}\right)^{2}+\frac{m_{v}^{2}}{\lambda^{2}} V_{\lambda}^{2}\right]\right. \\
& +\frac{1}{2} \lambda\left[\left(\nabla \phi_{\lambda}\right)^{2}+\frac{m_{s}^{2}}{\lambda^{2}} \phi_{\lambda}^{2}\right] \\
& \left.+\frac{b \phi_{\lambda}^{3}}{3 \lambda}+\frac{c \phi_{\lambda}^{4}}{4 \lambda}-\lambda^{2} e_{\infty}\left(\lambda^{3} \rho_{0}\right) \rho\right\}
\end{aligned}
$$

where all densities and fields depend on the variable $\lambda z$. With the help of the Klein-Gordon equations for the scaled vector and scalar fields derived from Eq. (10), the scaled surface tension can be recast as

$$
\begin{aligned}
\sigma_{\lambda}= & \int d(\lambda z)\left\{\lambda^{3} \widetilde{\mathcal{E}}_{\lambda}+\frac{1}{2} \lambda^{2} g_{v} V_{\lambda} \rho+\frac{1}{2} \lambda^{2} g_{s} \phi_{\lambda} \widetilde{\rho}_{s}\right. \\
& \left.-\frac{b \phi_{\lambda}^{3}}{6 \lambda}-\frac{c \phi_{\lambda}^{4}}{4 \lambda}-\lambda^{2} e_{\infty}\left(\lambda^{3} \rho_{0}\right) \rho\right\} .
\end{aligned}
$$

Using the explicit RTF or RETF expressions for the nuclear part of the energy and for the scalar density [20-23] together with the Klein-Gordon equations for $V_{\lambda}, \phi_{\lambda}, \partial V_{\lambda} / \partial \lambda$, and $\partial \phi_{\lambda} / \partial \lambda$ derived from Eq. (10), after some algebra the first and second derivatives of the scaled surface tension $\sigma_{\lambda}$ with respect to $\lambda$ at $\lambda=1$ read (see Refs. [20,21] for more details)

$$
\begin{aligned}
\left.\frac{d \sigma_{\lambda}}{d \lambda}\right|_{\lambda=1}= & 2 \sigma+\int_{-\infty}^{+\infty} d z\left\{\mathcal{E}-\rho_{s} m^{*}-m_{s}^{2} \phi^{2}-\frac{1}{2} g_{s} \rho_{s} \phi\right. \\
& \left.-\frac{1}{2} b \phi^{3}-\frac{1}{4} c \phi^{4}+\frac{1}{2} g_{v} \rho V+m_{v}^{2} V^{2}\right\}=0
\end{aligned}
$$

and 
TABLE I. Values of $\ddot{\sigma}$ (in $\mathrm{MeV} \mathrm{fm}{ }^{4}$ ) and $K_{s f}$ (in $\mathrm{MeV}$ ) calculated with the RTF and RETF approaches and the scaling method for several parameter sets. The nuclear matter incompressibility modulus $K_{\infty}$ (in $\mathrm{MeV}$ ) and the $-K_{s f} / K_{\infty}$ ratio are also listed.

\begin{tabular}{lcccccccc}
\hline \hline & & \multicolumn{3}{c}{ RTF } & \multicolumn{4}{c}{ RETF } \\
\cline { 3 - 5 } \cline { 7 - 8 } & $K_{\infty}$ & $\ddot{\sigma}$ & $K_{s f}$ & $-K_{s f} / K_{\infty}$ & $\ddot{\sigma}$ & $K_{s f}$ & $-K_{s f} / K_{\infty}$ \\
\hline NL-Z2 & 172.2 & -113.9 & -85.2 & 0.49 & -131.2 & -182.5 & 1.06 \\
NL1 & 211.1 & -170.3 & -170.6 & 0.81 & -171.8 & -225.4 & 1.07 \\
NL3 & 271.5 & -224.2 & -310.4 & 1.14 & -209.3 & -313.7 & 1.16 \\
NL-RA1 & 285.3 & -235.5 & -335.4 & 1.18 & -216.6 & -326.7 & 1.15 \\
NL-SH & 355.0 & -292.7 & -469.8 & 1.32 & -258.2 & -429.6 & 1.21 \\
NL2 & 399.2 & -295.9 & -521.0 & 1.31 & -279.0 & -482.8 & 1.21 \\
HS & 546.8 & -521.5 & -996.7 & 1.82 & -424.9 & -804.2 & 1.47 \\
L1 & 625.6 & -422.6 & -1024.6 & 1.64 & -320.6 & -787.1 & 1.26 \\
\hline \hline
\end{tabular}

$$
\begin{aligned}
\left.\frac{d^{2} \sigma_{\lambda}}{d \lambda^{2}}\right|_{\lambda=1}= & -6 \sigma+\int_{-\infty}^{+\infty} d z\left\{b \phi^{3}-\left.\left(b \phi^{2}+2 m_{s}^{2} \phi\right) \frac{\partial \phi_{\lambda}}{\partial \lambda}\right|_{\lambda=1}\right. \\
& +3 m_{s}^{2} \phi^{2}+\left.2 m_{v}^{2} V \frac{\partial V_{\lambda}}{\partial \lambda}\right|_{\lambda=1}-3 m_{v}^{2} V^{2} \\
& \left.+m \frac{\delta \rho_{s}}{\delta m^{*}}\left(m^{*}+\left.g_{s} \frac{\partial \phi_{\lambda}}{\partial \lambda}\right|_{\lambda=1}\right)-K_{\infty} \rho\right\} .
\end{aligned}
$$

The first derivative of $e_{\infty}\left(\lambda^{3} \rho_{0}\right)$ at $\lambda=1$ is just three times the pressure calculated at saturation density and thus it vanishes, while the second derivative gives $K_{\infty} \rho[21,26]$. On the other hand, since in the self-consistent RTF and RETF calculations the inputs for computing Eqs. (12) and (13) are quantities obtained from the solution of the variational equations associated with the surface tension (10) at $\lambda=1$, the so-called "sigma dot" theorem is rigorously fulfilled [27]. The method, therefore, allows $\ddot{\sigma}$ and consequently $K_{s f}$ to be computed on top of a self-consistent RTF or RETF calculation of the surface tension in symmetric semi-infinite nuclear matter. This is similar to what happens in the nonrelativistic frame with Skyrme forces [14], although in the relativistic case additional Klein-Gordon equations for $\partial V_{\lambda} / \partial \lambda$ and $\partial \phi_{\lambda} / \partial \lambda$ at $\lambda=1$ have to be solved.

Now we shall discuss the results obtained from the selfconsistent RTF and RETF methods in the scaling approximation. Table I collects $K_{\infty}, \ddot{\sigma}$, and $K_{s f}$ for the nonlinear NL-Z2 [28], NL1 [29], NL3 [30], NL-RA1 [31], NL-SH [32], and NL2 [33] and the linear HS [34] and L1 [33] parameter sets. One observes that in both the RTF and RETF calculations $\ddot{\sigma}$ and $K_{s f}$ decrease (become more negative) with increasing bulk incompressibility $K_{\infty}$. The RTF and RETF values of $\ddot{\sigma}$ and $K_{s f}$ for a given parameter set are, in general, rather different from one another, which means that the precise value of these quantities is model dependent. This is known to happen also with other quantities related with the nuclear surface. For example, such is the case of the surface energy coefficient of the leptodermous expansion of the binding energy of a nucleus, which is calculated as $4 \pi r_{0}^{2} \sigma$. The quality of the RTF and RETF approximations for semi-infinite nuclear matter and finite nuclei with respect to the RMF Hartree approach, and its dependence on the effective interaction, was investigated in Refs. [22,24,25] by analyzing the results obtained with many different parameter sets. It was noticed that the RTF results fluctuate around the corresponding Hartree results. Due to this fact there exist parametrizations for which the RTF approximation agrees by chance with the Hartree result. The behavior of RETF results in comparison with the Hartree solutions was found to be less dependent on the parameters of the force than in the RTF case, and it turned out that on the average the RETF results are in better agreement with the Hartree ones $[22,24,25]$.

The first contribution to $K_{s f}$ in Eq. (3) comes from the surface tension, let us call it $K_{s f}^{\sigma}$. The deviation found in the value of the surface tension from RTF calculations with respect to the corresponding RMF Hartree calculations is strongly correlated with the value of the effective mass in nuclear matter $m_{\infty}^{*} / m[22,25]$. For small values of $m_{\infty}^{*} / m$ the RTF surface tension is larger than the Hartree one, it practically agrees with the Hartree result for $m_{\infty}^{*} / m \sim 0.65$, and it becomes smaller than the Hartree result for larger $m_{\infty}^{*} / m$. On the other hand, the RETF result for the surface tension is consistently lower than the Hartree result and much less dependent on the specific value of $m_{\infty}^{*} / m$. (A similar situation is found for the total energy of finite nuclei $[22,24,25]$.) These trends, of course, are also reflected in $K_{s f}^{\sigma}$. For example, for NL1 $\left(m_{\infty}^{*} / m=0.57\right)$ we have $K_{s f}^{\sigma}=402.6,377$, and $429.3 \mathrm{MeV}$ in the Hartree, RETF, and RTF approaches, respectively. For NL2 $\left(m_{\infty}^{*} / m=0.67\right)$ it is $K_{s f}^{\sigma}=479.6,439.1$, and $465.7 \mathrm{MeV}$ in the Hartree, RETF and RTF calculation, respectively.

The second contribution to $K_{s f}$ in Eq. (3) is due to the second derivative of the surface tension. The results for $\ddot{\sigma}$ in the RTF approach decrease with $K_{\infty}$ faster than in the RETF calculation. At small values of $K_{\infty}$ the RTF value of $\ddot{\sigma}$ is less negative than that computed in the RETF approach, while the opposite happens for higher values of $K_{\infty}$. Both approaches predict the same value of $\ddot{\sigma}$ for an incompressibility around that of NL1 $(211 \mathrm{MeV})$. A similar behavior is dis- 
played by the self-consistent values of $K_{s f}$, although the crossing point between the RTF and RETF predictions is shifted to a larger value of $K_{\infty}$ (around $280 \mathrm{MeV}$ ) due to the fact that the contribution proportional to $\sigma\left(K_{s f}^{\sigma}\right)$ is larger in the RTF approach than in the RETF approach for the parameter sets considered here.

The $\ddot{\sigma}$ values obtained from the pocket formula Eq. (7) using the surface diffuseness of the RTF or RETF semiinfinite nuclear matter density profiles also decrease with $K_{\infty}$, though the estimate provided by Eq. (7) does not reproduce, in general, the self-consistent values very precisely. The approximate $\ddot{\sigma}$ is always smaller when calculated from the RTF approach than from the RETF approach for the parametrizations of Table I. Using Eq. (7) to estimate the value of $K_{s f}$ in first approximation, one finds that the RETF result is closer to the Hartree value than the RTF result for the sets NL-Z2, NL1, NL3, and NL-RA1. For NL2 and L1 it is the RTF estimate that lies closer to the Hartree estimate. For NL-SH and HS the approximate Hartree prediction lies roughly in between of the approximate RTF and RETF values. To the extent that Eq. (7) is applicable, it provides a hint of where the unknown exact Hartree value for $K_{s f}$ should lie with respect to the self-consistent RTF and RETF results presented in Table I.

Another different approach to computing $K_{s f}$ was proposed in Refs. [16,17]. It is based on the scaling method together with a local density approximation for the meson fields within the RTF approach. In Ref. [17] a $K_{s f}$ of approximately $-1000 \mathrm{MeV}$ was reported for a linear set with $K_{\infty}=545 \mathrm{MeV}$ (similar to the HS set). This result is in good agreement with that of our self-consistent RTF calculation for the HS set, though it is clearly larger in absolute value than the RETF result for $K_{s f}$ obtained with the HS set. On the other hand, our self-consistent RTF and RETF surface incompressibilities differ considerably from the estimate of Ref. [35] where approximate $K_{s f}$ values of -333.1 and $-610.1 \mathrm{MeV}$ were reported for the NL1 and NL-SH parametrizations calculated with the method used in Ref. [16].

It should also be pointed out that in our self-consistent semiclassical calculations we find that the ratio between the surface and bulk incompressibilities increases with $K_{\infty}$ (in agreement with the results of Ref. [17]). In the RETF case this ratio is close to one, as happens for the nonrelativistic Skyrme forces [5], provided that the bulk incompressibility $K_{\infty}$ of the interaction is not excessively high. In the RTF case the ratio between the surface and bulk incompressibilities increases much faster with $K_{\infty}$ than in the RETF calculations, and it considerably differs from unity for parametrizations with either a very low or a very high bulk incompressibility. In Fig. 1 we plot $-K_{s f}$ as a function of $K_{\infty}$ for the parameter sets considered in Table I. As in the nonrelativistic case [2], $K_{s f}$ varies roughly linearly with $K_{\infty}$. A linear fit of all the points gives $-K_{s f}=1.47 K_{\infty}-84$ in the RETF model and $-K_{s f}=2.19 K_{\infty}-295$ in the RTF model. If only the nonlinear parametrizations are included in the fit one obtains $-K_{s f}=1.35 K_{\infty}-54$ and $-K_{s f}=1.96 K_{\infty}-238$ in the RETF and RTF cases, respectively.

The surface incompressibility coefficient is both large and

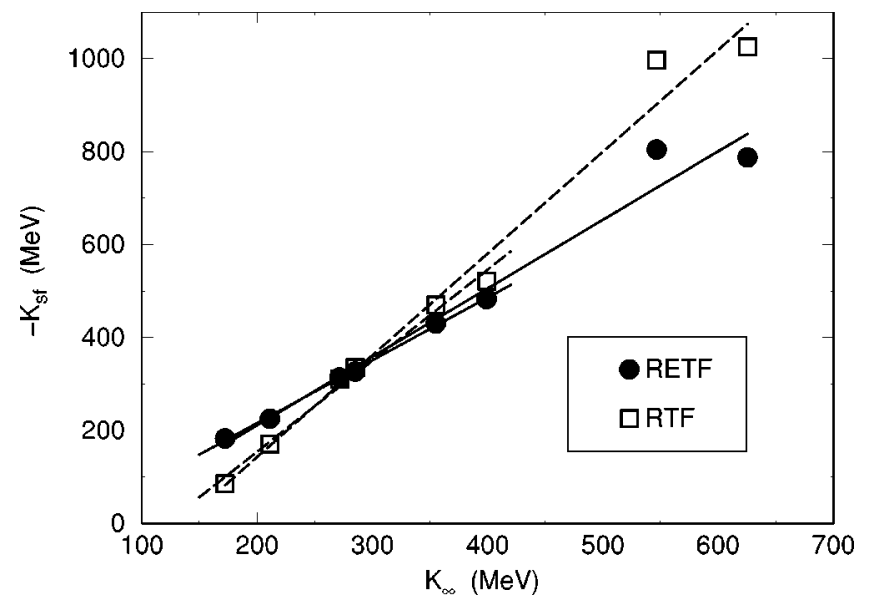

FIG. 1. Surface incompressibility coefficient versus the nuclear matter incompressibility modulus for the parameter sets of Table I.

negative, thus its contribution considerably reduces the finite nucleus incompressibility $K_{A}$ with respect to the nuclear matter limit $K_{\infty}$. This result, although obtained in the scaling model, illustrates the physical effect that the compression of the surface provides a considerable reduction of $K_{A}$, which is also found in more fundamental RPA calculations [6]. In Ref. [21] we have self-consistently computed the finite nucleus incompressibility $K_{A}$ using the RETF approach and the scaling method that we have employed in the present work to obtain $K_{s f}$. Thus we can now precisely check the ability of the leptodermous expansion Eq. (2) in reproducing the full calculation of $K_{A}$ carried out in Ref. [21] for various finite nuclei.

The coefficients $K_{v s}$ and $K_{\text {coul }}$ entering Eq. (2) are computed using nuclear matter properties only. Explicit expressions for these coefficients in the nonlinear $\sigma-\omega$ model are reported in Ref. [18]. In our analysis we will use the NL1, NL3, and NL-SH parameter sets for which the numerical values of these coefficients are given in Ref. [19]. The surface incompressibility coefficient is the self-consistent value taken from Table I. Table II collects $K_{A}$ obtained from the

TABLE II. Finite nuclei incompressibilities (in $\mathrm{MeV}$ ) calculated with the self-consistent RETF approach $\left(K_{A}\right)$ and with the leptodermous expansion Eq. (2) $[K(A, I)]$. Results are presented for the NL1, NL3, and NL-SH parameter sets.

\begin{tabular}{lcccccc}
\hline \hline & \multicolumn{2}{c}{ NL1 } & \multicolumn{2}{c}{ NL3 } & \multicolumn{2}{c}{ NL-SH } \\
\hline & $K_{A}$ & $K(A, I)$ & $K_{A}$ & $K(A, I)$ & $K_{A}$ & $K(A, I)$ \\
\hline${ }^{40} \mathrm{Ca}$ & 108.2 & 128.1 & 145.3 & 161.0 & 196.8 & 208.6 \\
${ }^{48} \mathrm{Ca}$ & 111.1 & 116.9 & 147.4 & 151.0 & 198.3 & 198.4 \\
${ }^{56} \mathrm{Ni}$ & 115.0 & 130.8 & 153.2 & 166.0 & 207.1 & 216.7 \\
${ }^{90} \mathrm{Zr}$ & 122.5 & 129.3 & 161.6 & 167.3 & 217.5 & 221.1 \\
${ }^{116} \mathrm{Sn}$ & 124.3 & 126.3 & 163.4 & 165.4 & 219.8 & 220.4 \\
${ }^{132} \mathrm{Sn}$ & 121.3 & 105.4 & 157.6 & 144.9 & 210.9 & 197.5 \\
${ }^{144} \mathrm{Sm}$ & 125.4 & 125.3 & 164.5 & 165.3 & 221.6 & 221.5 \\
${ }^{208} \mathrm{~Pb}$ & 124.1 & 111.1 & 161.1 & 152.1 & 216.7 & 208.1 \\
\hline \hline
\end{tabular}




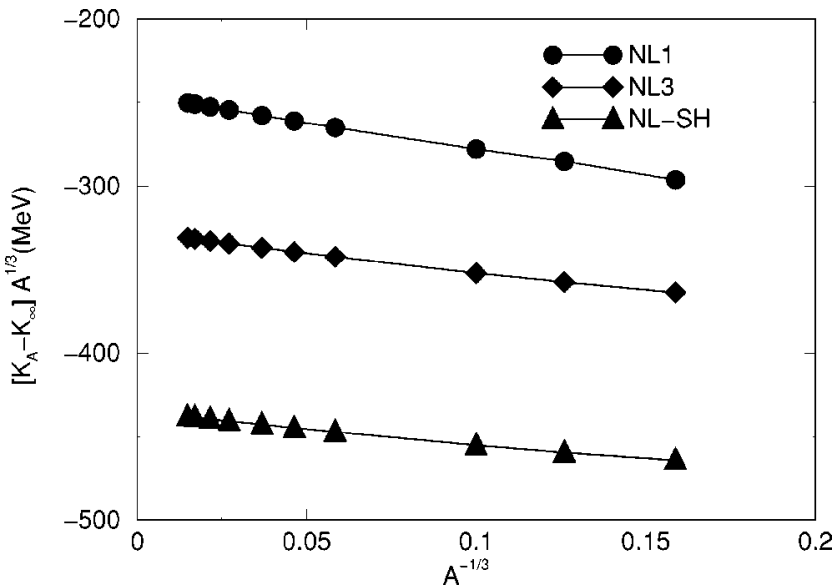

FIG. 2. $\left(K_{A}-K_{\infty}\right) A^{1 / 3}$ versus $A^{-1 / 3}$ computed for several uncharged and symmetric nuclei from $A=250$ to $A=300000$ for the NL1, NL3, and NL-SH parameter sets.

full self-consistent RETF calculation [21] as well as the value $K(A, I)$ given by Eq. (2) for ${ }^{40} \mathrm{Ca},{ }^{48} \mathrm{Ca},{ }^{56} \mathrm{Ni},{ }^{90} \mathrm{Zr}$, ${ }^{116} \mathrm{Sn},{ }^{132} \mathrm{Sn},{ }^{144} \mathrm{Sm}$, and ${ }^{208} \mathrm{~Pb}$. From this table it can be seen that the leptodermous expansion with the terms given in Eq. (2) fails to describe small nuclei and also very asymmetric nuclei such as ${ }^{132} \mathrm{Sn}$ or ${ }^{208} \mathrm{~Pb}$. In addition, some words of caution should be said about the Coulomb term in Eq. (2). In the self-consistent scaling calculation of the finite nucleus incompressibility, the Coulomb energy does not participate directly if the scaling Eq. (5) for the density is assumed to apply $[3,21]$. Thus, the Coulomb term in Eq. (2) should be related to the change in $K_{A}$ when the Coulomb interaction is switched off in the self-consistent calculation. The Coulomb term in Eq. (2) overestimates this change by approximately 6 $\mathrm{MeV}$ for NL1, $3 \mathrm{MeV}$ for NL3, and $1 \mathrm{MeV}$ for NL-SH.

Now we would like to analyze whether the addition of some higher-order terms in the leptodermous expansion Eq. (2) improves the agreement with the $K_{A}$ results calculated self-consistently. In particular, we will focus our attention on the curvature $K_{c v} A^{-1 / 3}$ and the surface-symmetry $K_{s s} I^{2} A^{-1 / 3}$ terms. Although these terms could be derived by enlarging the leptodermous expansion of Blaizot [2], as has been done

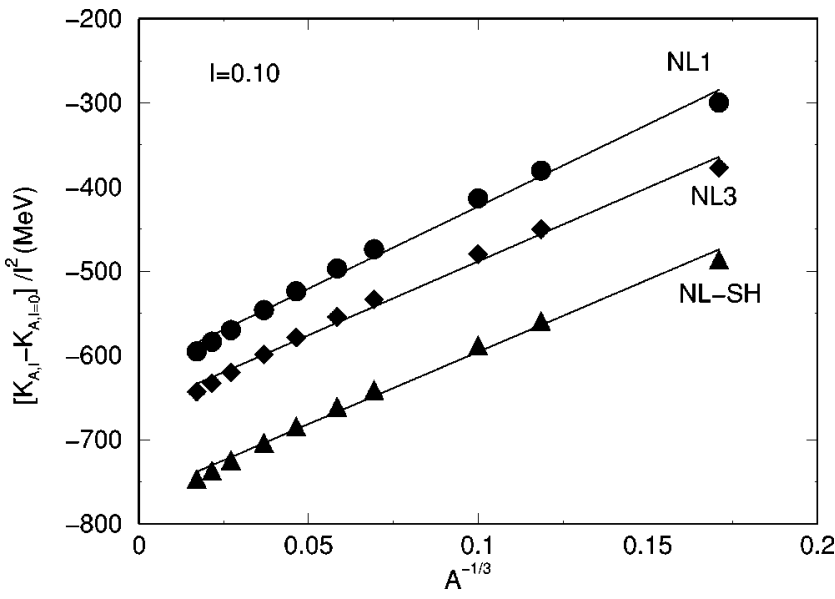

FIG. 3. $\left(K_{A, I}-K_{A, I=0}\right) / I^{2}$ versus $A^{-1 / 3}$ for several uncharged nuclei from $A=200$ to $A=200000$ with a neutron excess 0.10 for the NL1, NL3, and NL-SH parameter sets.

in the nonrelativistic case [12], it becomes more complicated in the relativistic case. Thus, for a fast estimate of the curvature and surface-symmetry terms, we perform a numerical fit. To do this, we follow the same strategy as in Ref. [12]. First we consider symmetric nuclei with the Coulomb force switched off. In this case the leptodermous expansion Eq. (2) (adding the curvature term) reduces to

$$
K_{A}=K_{\infty}+K_{s f} A^{-1 / 3}+K_{c v} A^{-2 / 3} .
$$

In Fig. 2 we plot $\left[K_{A}-K_{\infty}\right] A^{1 / 3}$ versus $A^{-1 / 3}$ for the three parameters sets used in this analysis. Here $K_{\infty}$ is the nuclear matter incompressibility given in Table I and $K_{A}$ are the self-consistent incompressibilities calculated for $A$ ranging from 250 to 300000 . In the linear fit of these curves the $y$-axis intercept gives $K_{s f}$ of the corresponding force, while the slope gives $K_{c v}$. The surface terms obtained in this way are $-246.1,-328.4$, and $-435.8 \mathrm{MeV}$ for the NL1, NL3, and NL-SH parameter sets, which are very close to the corresponding self-consistent values (see Table I). The estimates of the curvature term in the leptodermous expansion of the

TABLE III. Finite nuclei incompressibilities (in $\mathrm{MeV}$ ) for several uncharged nuclei calculated selfconsistently using the RETF approach $\left(K_{A}\right)$, with the leptodermous expansion Eq. (2) $[K(A, I)]$ and including the curvature and surface-symmetry contributions $\left[K^{*}(A, I)\right]$. Results are presented for the NL1, NL3, and NL-SH parameter sets.

\begin{tabular}{lccccccccc}
\hline \hline & \multicolumn{3}{c}{ NL1 } & \multicolumn{3}{c}{ NL3 } & \multicolumn{3}{c}{ NL-SH } \\
\hline & $K_{A}$ & $K(A, I)$ & $K^{*}(A, I)$ & $K_{A}$ & $K(A, I)$ & $K^{*}(A, I)$ & $K_{A}$ & $K(A, I)$ & $K^{*}(A, I)$ \\
\hline${ }^{40} \mathrm{Ca}$ & 118.6 & 145.2 & 118.1 & 160.1 & 179.8 & 160.2 & 213.4 & 229.4 & 213.5 \\
${ }^{48} \mathrm{Ca}$ & 119.6 & 130.3 & 121.2 & 159.7 & 165.8 & 161.8 & 215.1 & 214.7 & 213.8 \\
${ }^{56} \mathrm{Ni}$ & 129.3 & 152.2 & 130.5 & 172.7 & 189.6 & 173.9 & 230.5 & 242.7 & 230.0 \\
${ }^{90} \mathrm{Zr}$ & 139.6 & 152.5 & 142.0 & 184.2 & 192.9 & 186.3 & 244.3 & 249.3 & 244.8 \\
${ }^{116} \mathrm{Sn}$ & 144.0 & 152.0 & 146.3 & 189.0 & 193.9 & 191.1 & 250.1 & 251.8 & 250.7 \\
${ }^{132} \mathrm{Sn}$ & 137.2 & 127.1 & 137.4 & 179.3 & 168.9 & 180.2 & 236.8 & 223.9 & 236.58 \\
${ }^{144} \mathrm{Sm}$ & 148.5 & 155.0 & 150.7 & 194.3 & 198.2 & 196.3 & 256.7 & 257.7 & 257.2 \\
${ }^{208} \mathrm{~Pb}$ & 148.4 & 142.8 & 148.5 & 193.4 & 187.3 & 194.0 & 255.0 & 246.9 & 254.6 \\
\hline \hline
\end{tabular}


finite nucleus incompressibility obtained with NL1, NL3, and NL-SH are $-317.2,-229.8$ and $-185.6 \mathrm{MeV}$, respectively.

To obtain the surface-symmetry contribution, we have found it convenient to parametrize the difference between the self-consistent incompressibilities $K_{A}$ of a given nucleus with neutron excess $I$ and the corresponding symmetric nucleus as

$$
K_{A, I}-K_{A, I=0}=K_{v s} I^{2}+K_{s s} I^{2} A^{-1 / 3},
$$

where again uncharged nuclei have been considered. For each parameter set and according to Eq. (15), if $\left[K_{A, I}\right.$ $\left.-K_{A, I=0}\right] I^{-2}$ is plotted versus $A^{-1 / 3}$ a unique curve should be found which is independent of the value of $I$. However, one obtains a family of almost parallel lines whose slope is $K_{s s}$. The splitting of these lines gives us information on the higher-order symmetry contributions missed in the parametrization (15). Thus we will estimate the surface-symmetry term from a linear fit of the curve corresponding to $I=0.1$, which roughly corresponds to an average asymmetry along the periodic table. This curve is plotted in Fig. 3 for $A$ ranging from 200 to 200000 for each considered parameter set. The corresponding $y$-axis intercepts agree very well with the $K_{v s}$ values calculated in nuclear matter $(-676.1,-698.9$, and $-794.5 \mathrm{MeV}$ for NL1, NL3, and NL-SH respectively [19]). Our estimate of the surface-symmetry contribution to $K_{A}$ corresponds to the slopes of these linear fits, which are 1951.4, 1754.0, and 1716.5 MeV for NL1, NL3, and NL-SH, respectively.

Table III collects the self-consistent finite nuclei incompressibility $K_{A}$ (without Coulomb) compared with the macroscopic parametrizations $K(A, I)[$ Eq. $(2)]$ and $K^{*}(A, I)$ that contains the curvature and surface-symmetry contributions obtained from the previously discussed fits. Again, the selfconsistent incompressibilities corresponding to the lightest nuclei and the very asymmetric nuclei are not well reproduced by the simplest expansion Eq. (2). If the curvature and surface-symmetry corrections are included, the improved macroscopic formula $K^{*}(A, I)$ reproduces the self-consistent incompressibilities with an error, on average, smaller than $1.2 \%, 0.9 \%$, and $0.3 \%$ for the NL1, NL3, and NL-SH parameter sets. In order to gain some insight into the accuracy of our estimate of the curvature and surface-symmetry con- tributions, we fit the self-consistent results for the finite nuclei considered in Table III to a leptodermous expansion including curvature and surface-symmetry terms. The volume, surface, and volume-symmetry coefficients are taken from self-consistent infinite and semi-infinite nuclear matter calculations. The results of this calculation show that the difference of the curvature contribution obtained from the fit in the asymptotic region and from finite nuclei is always less than $10 \%$, whereas the difference in the surface-symmetry contribution lies below $3 \%$.

We have applied the scaling method in the Thomas-Fermi and extended Thomas-Fermi approximations to the relativistic mean-field theory to self-consistently calculate the surface coefficient $K_{s f}$ of the leptodermous expansion of the finite nucleus incompressibility derived within the Blaizot model. The ratio between the surface and bulk incompressibilities obtained in our semiclassical calculation increases with the nuclear matter incompressibility, more strongly in the RTF than in the RETF case. In the RETF calculations this ratio is close to one, as in the case of non-relativistic Skyrme forces, for the nonlinear parameter sets that have a nuclear matter incompressibility not larger than roughly $300 \mathrm{MeV}$.

For the analyzed $\sigma-\omega$ parameter sets, the leptodermous expansion Eq. (2) is not able to reproduce very precisely the finite nuclei incompressibilities obtained self-consistently. In particular, the macroscopic contribution of the Coulomb force can differ from the self-consistent contribution up to 6 $\mathrm{MeV}$. We have numerically estimated higher-order contributions to the leptodermous expansion, namely, curvature and surface-symmetry terms, in the asymptotic region (i.e., for very large uncharged systems). We have found that the finite nuclei incompressibilities are reasonably well reproduced by an extended leptodermous expansion that includes curvature and surface-symmetry contributions.

Useful discussions with E. Vives, M. Farine, and J.N. De are acknowledged. The authors would like to acknowledge support from the DGICYT (Spain) under Grant No. PB981247 and from DGR (Catalonia) under Grant No. 2000SGR00024. S.K.P. thanks the Spanish Education Ministry Grant No. SB97-OL174874 for financial support and the Departament d'Estructura i Constituents de la Materia of the University of Barcelona for kind hospitality.
[1] P. Ring and P. Schuck, The Nuclear Many-Body Problem (Springer-Verlag, Berlin, 1980).

[2] J.P. Blaizot, Phys. Rep. 64, 171 (1980).

[3] O. Bohigas, A. Lane, and J. Martorell, Phys. Rep. 51, 267 (1979)

[4] B.K. Jennings and A.D. Jackson, Phys. Rep. 66, 141 (1980).

[5] J. Treiner, H. Krivine, O. Bohigas, and J. Martorell, Nucl. Phys. A371, 253 (1981).

[6] J.P. Blaizot, J.F. Berger, J. Dechargé, and M. Girod, Nucl. Phys. A591, 435 (1995).

[7] M. Farine, J.M. Pearson, and F. Tondeur, Nucl. Phys. A615, 135 (1997).
[8] Zhongyu Ma, Nguyen Van Giai, A. Wandelt, D. Vretenar, and P. Ring, Nucl. Phys. A686, 173 (2001).

[9] M.M. Sharma, W.T.A. Borghols, S. Brandeburg, S. Crona, A. Van der Woude, and M.N. Harakeh, Phys. Rev. C 38, 2562 (1988); M.M. Sharma, W. Stocker, P. Gleissl, and M. Brack, Nucl. Phys. A504, 337 (1989).

[10] J.M. Pearson, Phys. Lett. B 271, 1 (1991).

[11] S. Shlomo and D.H. Youngblood, Phys. Rev. C 47, 529 (1993).

[12] R.C. Nayak, J.M. Pearson, M. Farine, P. Gleissl, and M. Brack, Nucl. Phys. A516, 62 (1990).

[13] W.D. Myers and W.J. Swiatecki, Ann. Phys. (N.Y.) 55, 395 (1969). 
[14] J.M. Pearson, M. Farine, and J. Côté, Phys. Rev. C 26, 267 (1982).

[15] M. Brack and W. Stocker, Nucl. Phys. A388, 320 (1982); A406, 413 (1983).

[16] W. Stocker and M.M. Sharma, Z. Phys. A 339, 147 (1991).

[17] M.M. Sharma, M.A. Nagarajan, and P. Ring, Ann. Phys. (N.Y.) 231, 110 (1994).

[18] D. Von-Eiff, J.M. Pearson, W. Stocker, and M.K. Weigel, Phys. Rev. C 50, 831 (1994).

[19] T.v. Chossy and W. Stocker, Phys. Rev. C 56, 2518 (1997).

[20] S.K. Patra, M. Centelles, X. Viñas, and M. Del Estal, Phys. Lett. B 523, 67 (2001).

[21] S.K. Patra, X. Viñas, M. Centelles, and M. Del Estal, Nucl. Phys. A (to be published).

[22] M. Centelles, X. Viñas, M. Barranco, and P. Schuck, Ann. Phys. (N.Y.) 221, 165 (1993); M. Centelles and X. Viñas, Nucl. Phys. A563, 173 (1993); M. Del Estal, M. Centelles, and X. Viñas, Phys. Rev. C 56, 1774 (1997).

[23] M. Centelles, M. Del Estal, and X. Viñas, Nucl. Phys. A635, 193 (1998).

[24] M. Centelles, X. Viñas, M. Barranco, S. Marcos, and R.J.
Lombard, Nucl. Phys. A537, 486 (1992).

[25] C. Speicher, E. Engel, and R.M. Dreizler, Nucl. Phys. A562, 569 (1993).

[26] S. Nishizaki, H. Kurasawa, and T. Suzuki, Nucl. Phys. A462, 689 (1987).

[27] M. Farine and W. Stocker, Nucl. Phys. A454, 71 (1986).

[28] M. Bender, K. Rutz, P.-G. Reinhard, J.A. Maruhn, and W. Greiner, Phys. Rev. C 60, 034304 (1999).

[29] P.-G. Reinhard, M. Rufa, J. Maruhn, W. Greiner, and J. Friedrich, Z. Phys. A 323, 13 (1986).

[30] G.A. Lalazissis, J. König, and P. Ring, Phys. Rev. C 55, 540 (1997).

[31] M. Rashdan, Phys. Rev. C 63, 044303 (2001).

[32] M.M. Sharma, M.A. Nagarajan, and P. Ring, Phys. Lett. B 312, 377 (1993).

[33] S.J. Lee, J. Fink, A.B. Balantekin, M.R. Strayer, A.S. Umar, P.-G. Reinhard, J.A. Maruhn, and W. Greiner, Phys. Rev. Lett. 57, 2916 (1986).

[34] C.J. Horowitz and B.D. Serot, Nucl. Phys. A368, 503 (1981).

[35] M.V. Stoitsov, M.L. Cescato, P. Ring, and M.M. Sharma, J. Phys. G 20, L149 (1994). 